\title{
Using Spherical Moments for Visual Servoing from a Special Target with Unique Projection Center Cameras
}

\author{
Romeo Tatsambon Fomena and François Chaumette \\ IRISA - Université de Rennes 1 and INRIA \\ Rennes, France \\ firstname.name@irisa.fr
}

\begin{abstract}
This paper focuses on visual servoing from a special target using a vision sensor with an unique projection center. The target is a sphere marked with two points on its surface. Using a spherical projection model, a new minimal set of six features is proposed for this target. Spherical moments are exploited to compute the new set on the image of the sensor. Using the new set, a classical control method is proved to be asymptotically stable even in the presence of modeling errors. Experimental results with a perspective camera and a fish-eye camera validate the proposed theoretical results.
\end{abstract}

Index Terms-Visual servoing, central projection cameras, spherical moments.

\section{INTRODUCTION}

In visual servoing, data provided by a vision sensor is used to control the motion of a robot [1]. A vision sensor provides a large spectrum of possible visual features. However, using some of the features could lead to potential problems of stability or visibility if the robot has to achieve a very large displacement [2]. For this reason, we need to choose ideal visual features for visual servoing. By ideal, satisfaction of the following criteria is meant: local and -as far as possibleglobal stability of the system, robustness to calibration and to modeling errors, non-singularity, local minima avoidance, satisfactory motion of the system and of the features in the image, and finally maximal decoupling and linear link (the ultimate goal) between the visual features and the degrees of freedom (DOFs) taken into account. In this context, several methods have been proposed to approach an ideal system behaviour using either 3D data [3], [4] and [5]; hybrid (3D and 2D) data [6]; or 2D data [7], [8] and [9].

Vision sensors can be classified in two groups: the ones satisfying the unique viewpoint constraint also called central projection cameras and the others. The first class comprises perspective cameras and central catadioptric systems which combine a camera and a mirror to enlarge their field of view (FOV) [10]. The second class comprises fish-eye cameras which use a short focal length to widen their FOV. Central projection cameras share the spherical projection as it has been proven in [11]. Similarly, it is possible to approximate a map between a fish-eye image and a sphere using an accurate projection model [12]. Thus, the spherical projection model can be considered as a unified approach for modeling visual features with most omnidirectional cameras and also with perspective cameras.

A generic representation of the image of the target can be obtained using moments computed on the surface of the sphere, also known as spherical moments. These particular moments have been used recently to design a new decoupled scheme for visual servoing from points with any central projection camera [13]. For certain under-actuated systems, the use of a spherical projection model for visual servoing is suitable since this projection of an image point conserves the passivity property, this property being important to control such systems [14]. This projection model has been used in [15] to define a global diffeomorphism to control the six DOFs of a system using a particular target; in [15], this diffeomorphism has also been coupled with navigation functions to design a global controller in a well defined domain taking into account occlusions and FOV boundary. The target in that case was a sphere marked with a tangent vector to a point on its surface (which we refer to as CC target).

In this paper, we consider a more realistic special target composed of a sphere marked with two points on its surface (which we refer to as special sphere). Indeed, from a practical point of view the special sphere is more natural than the $\mathrm{CC}$ target. In addition the special sphere can be used as an alternative passive target for spacecraft autonomous docking as it has been done with three spheres in [16].

In this paper, using a simple sphere as a target, we recall that the translation part of the diffeomorphism proposed in [15] has been improved in [8]. In the next section, we build on the previous work on spheres and propose a new minimal set of six features for the special sphere. Using the new set of features, a theoretical analysis of the stability of a classical control law with respect (w.r.t.) to modeling error is given in Section III. In Section IV spherical moments are utilised to compute the new set. Few years ago, it was shown how to compute spherical moments for a perspective image plane [9]. Here, this computation is generalized to any central projection and fish-eye image planes. Finally, experimental results with perspective and fish-eye cameras are given in Section V. 


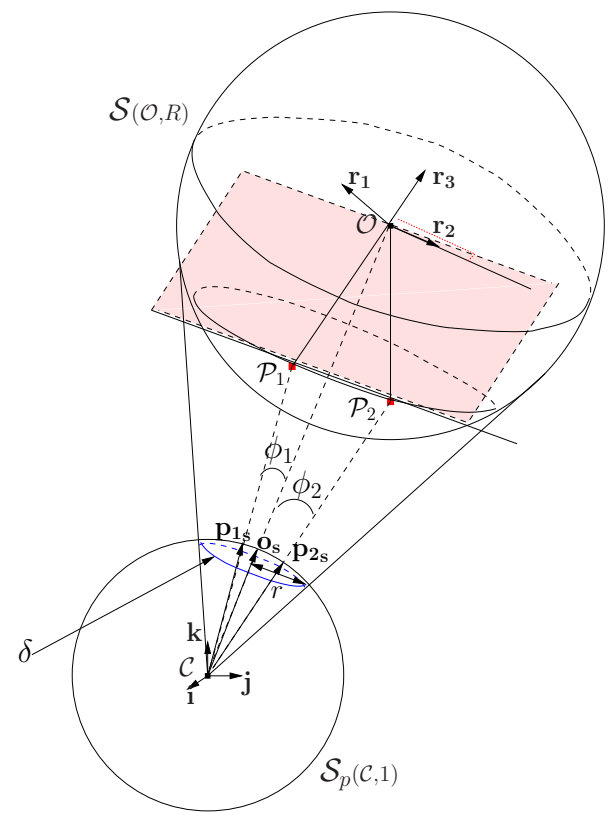

Fig. 1. Spherical projection of the special sphere.

\section{FEATURE MODELING USING A SPHERICAL PROJECTION MODEL}

In this section we first present the minimal set of three features for visual servoing from spheres lately proposed in [8]. We then propose a new minimal set for the special sphere. These two sets are obtained using a spherical projection model.

We recall that the interaction matrix $\mathbf{L}_{\mathbf{f}}$ related to a set of features $\mathbf{f} \in \mathbb{R}^{n}$ is defined such that $\dot{\mathbf{f}}=\mathbf{L}_{\mathbf{f}} \mathbf{v}_{c}$ where $\mathbf{v}_{c}=(\boldsymbol{v}, \boldsymbol{\omega}) \in \mathfrak{s e}(\mathbf{3})$ is the instantaneous camera velocity [17]: $\boldsymbol{v}$ and $\boldsymbol{\omega}$ are respectively the translational and the rotational velocities of the camera and $\mathfrak{s e}(\mathbf{3}) \simeq \mathbb{R}^{3} \times \mathbb{R}^{3}$ is the Lie algebra of the Lie group of displacements $\operatorname{SE}(3)$.

\section{A. Simple sphere}

Let $\mathcal{F}_{c}=(\mathcal{C}, \mathbf{i}, \mathbf{j}, \mathbf{k})$ be the center of projection frame and $\mathcal{S}_{p}(\mathcal{C}, 1)$ the unit sphere of projection. Let $\mathcal{S}(\mathcal{O}, R)$ be a sphere of radius $R$ and center $\mathcal{O}$. Let $\mathbf{O}$ be the vector coordinates of $\mathcal{O}$ in $\mathcal{F}_{c}$. The spherical projection of $\mathcal{S}(\mathcal{O}, R)$ is a dome hat [15]. This dome hat can be characterized by the circular contour $\delta$ of its base (see Fig. 1).

A set of four features has been proposed in [15] to control the image of the sphere: $\mathbf{s}_{\mathbf{p}}=\left(r, \mathbf{o}_{\mathbf{s}}\right)$ where

$$
r=\frac{R}{\|\mathbf{O}\|}
$$

is the radius of $\delta$ and

$$
\mathbf{o}_{\mathbf{s}}=\frac{\mathbf{O}}{\|\mathbf{O}\|}=\frac{1}{\|\mathbf{O}\|}{ }^{c} \mathbf{t}_{o}
$$

is the dome hat summit, with ${ }^{c} \mathbf{t}_{o}$ the relative position of $\mathcal{S}(\mathcal{O}, R)$ in $\mathcal{F}_{c}$.

This set is not minimal since three parameters are sufficient to describe the image of a sphere [17]. For this reason the minimal set of features

$$
\mathbf{s}=\frac{1}{r} \mathbf{o}_{\mathbf{s}}=\frac{1}{R}{ }^{c} \mathbf{t}_{o}
$$

has been proposed in [8]. The interaction matrix $\mathbf{L}_{\mathbf{s}}$ related to $\mathbf{s}$ is given by:

$$
\mathbf{L}_{\mathbf{s}}=\left[\begin{array}{ll}
-\frac{1}{R} \mathbf{I}_{3} & {[\mathbf{s}]_{\times}}
\end{array}\right],
$$

where $[\mathbf{s}]_{X}$ is the skew matrix related to $\mathbf{s}$. This corresponds to the interaction matrix related to the $3 \mathrm{D}$ point $\mathcal{O}$ up to the scale factor $R$. In addition to the decoupling property, $\mathbf{L}_{\mathbf{s}}$ presents the same dynamic $\left(\frac{1}{R}\right)$ in the translational velocities. Since $R$ is a constant, there is a linear link between the visual features and the camera translational velocities. Moreover we can note that the interaction matrix $\mathbf{L}_{\mathbf{s}}$ presents the passivity property, which is important to control certain under-actuated systems [14].

\section{B. Special sphere}

Inspired by the CC target introduced in [15], we use a special sphere which is obtained by gluing two feature points $\mathcal{P}_{1}$ and $\mathcal{P}_{2}$ on the surface of $\mathcal{S}(\mathcal{O}, R)$. Let ${ }^{c} \mathbf{R}_{o}=\left[\begin{array}{lll}\mathbf{r}_{1} & \mathbf{r}_{2} & \mathbf{r}_{3}\end{array}\right]$ be the relative orientation of the special sphere w.r.t. $\mathcal{F}_{c}$. Fig. 1 presents the spherical projection of the special sphere. Here we are interested in controlling the six DOFs of the system.

The three features $\mathbf{s}$ charaterizing the image of $\mathcal{S}(\mathcal{O}, R)$, as shown above, are used to control the camera position.

The control of the camera orientation is done by using the 3D features $\theta \mathbf{u}$ (where $\theta$ is the angle of rotation and $\mathbf{u}$ the axis of rotation) computed from the relative orientation ${ }^{c *} \mathbf{R}_{c}$ of the current frame w.r.t. the desired frame. We now show how to determine the orientation ${ }^{c} \mathbf{R}_{o}=\left[\begin{array}{lll}\mathbf{r}_{1} & \mathbf{r}_{2} & \mathbf{r}_{3}\end{array}\right]$ of the target w.r.t. the camera.

Features vector $\mathcal{P}_{1} \mathcal{P}_{2}$ of the special sphere is defined such that:

$$
\mathbf{a}_{12}=\mathbf{P}_{1} \mathbf{P}_{2}=\lambda_{1} \mathbf{r}_{3}+\lambda_{2} \mathbf{r}_{2}
$$

where $\left(\lambda_{1}, \lambda_{2}\right) \in \mathbb{R}^{2}$ and $\mathbf{P}_{1}$ (respectively $\mathbf{P}_{2}$ ) is the vector coordinates of the feature point $\mathcal{P}_{1}$ (respectively $\mathcal{P}_{2}$ ). It is shown in [15] that the feature point $\mathcal{P}_{1}$ is such that (see Fig. 1):

$$
\mathbf{r}_{\mathbf{3}}=\frac{1}{R}\left({ }^{c} \mathbf{t}_{o}-\mathbf{P}_{1}\right) \text {. }
$$

where $\frac{1}{R} \mathbf{P}_{1}=\frac{\left\|\mathbf{P}_{1}\right\|}{R} \mathbf{p}_{\mathbf{1}_{\mathbf{s}}}$ with $\mathbf{p}_{\mathbf{1}_{\mathbf{s}}}=\mathbf{P}_{1} /\left\|\mathbf{P}_{1}\right\|$. It is possible to compute $\left\|\mathbf{P}_{1}\right\| / R$ by applying the cosine rule to the triangle $\left(\mathcal{C}, \mathcal{P}_{1}, \mathcal{O}\right)$ in $\mathcal{F}_{c}[15]$. We obtain the second degree equation in $\left\|\mathbf{P}_{1}\right\|$

$$
\left\|\mathbf{P}_{1}\right\|^{2}+\|\mathbf{O}\|^{2}-2\left\|\mathbf{P}_{1}\right\|\|\mathbf{O}\| \cos \phi_{1}=R^{2}
$$

where $\phi_{1}=\mathbf{o}_{\mathbf{s}}^{\top} \mathbf{p}_{\mathbf{1}_{\mathbf{s}}}$. The two solutions depending on $\sigma= \pm 1$ are given by

$$
\left\|\mathbf{P}_{1}\right\|=\frac{R}{r}\left(\cos \phi_{1}+\sigma \sqrt{r^{2}-\sin ^{2} \phi_{1}}\right)
$$

from which it is easy to deduce $\left\|\mathbf{P}_{1}\right\| / R$. The choice of $\sigma=-1$ is related to the visibility condition defined in [15]. At this point we can conclude that $\mathbf{r}_{3}$ can be computed from 
the image of the special sphere. Indeed, since ${ }^{c} \mathbf{t}_{o} / R$ is nothing but $\mathbf{s}$, we obtain from (3) and (5)

$$
\mathbf{r}_{\mathbf{3}}=\mathbf{s}-\frac{1}{r}\left(\cos \phi_{1}-\sqrt{r^{2}-\sin ^{2} \phi_{1}}\right) \mathbf{p}_{\mathbf{1}_{\mathbf{s}}} .
$$

Likewise $\left\|\mathbf{P}_{1}\right\| / R$, we obtain

$$
\left\|\mathbf{P}_{2}\right\| / R=\left(\cos \phi_{2}-\sqrt{r^{2}-\sin ^{2} \phi_{2}}\right) / r
$$

where $\phi_{2}=\mathbf{o}_{\mathbf{s}}^{\top} \mathbf{p}_{\mathbf{2}_{\mathbf{s}}}$ with $\mathbf{p}_{\mathbf{2}_{\mathbf{s}}}=\mathbf{P}_{2} /\left\|\mathbf{P}_{2}\right\|$. It is therefore possible to compute

$$
\frac{1}{R} \mathbf{a}_{\mathbf{1 2}}=\frac{1}{R}\left(\mathbf{P}_{2}-\mathbf{P}_{1}\right),
$$

which can be expressed using (5) and (7) as

$$
\begin{aligned}
& \frac{1}{R} \mathbf{a}_{\mathbf{1 2}}=\frac{\left(\cos \phi_{1}-\sqrt{r^{2}-\sin ^{2} \phi_{1}}\right)}{r} \mathbf{p}_{\mathbf{1}_{\mathbf{s}}} \\
& -\frac{\left(\cos \phi_{2}-\sqrt{r^{2}-\sin ^{2} \phi_{2}}\right)}{r} \mathbf{p}_{\mathbf{2}_{\mathbf{s}}} .
\end{aligned}
$$

From (9) and (2) we obtain:

$$
\frac{\lambda_{2}}{R} \mathbf{r}_{\mathbf{2}}=\left(\frac{1}{R} \mathbf{a}_{\mathbf{1 2}}-\left(\left(\frac{1}{R} \mathbf{a}_{\mathbf{1 2}}\right)^{\top} \mathbf{r}_{\mathbf{3}}\right) \mathbf{r}_{\mathbf{3}}\right),
$$

from which we easily deduce $\mathbf{r}_{\mathbf{2}}$. Finally we have $\mathbf{r}_{\mathbf{1}}=\mathbf{r}_{\mathbf{2}} \times \mathbf{r}_{\mathbf{3}}$ which gives us ${ }^{c} \mathbf{R}_{o}$. Similarly, it is possible to compute ${ }^{c *} \mathbf{R}_{o}$ from the desired image and thus to obtain ${ }^{c *} \mathbf{R}_{c}$.

To conclude, it is possible to compute the partial pose $\left({ }^{c} \mathbf{t}_{o} / R, \theta \mathbf{u}\right)$ of the special sphere and therefore to select the minimal set of six features $\mathbf{s}_{\mathbf{n}}=(\mathbf{s}, \theta \mathbf{u})$ for visual servoing w.r.t. the special sphere. The interaction matrix related to $\mathbf{s}_{\mathbf{n}}$ has the following form

$$
\mathbf{L}_{\mathbf{s}_{\mathbf{n}}}=\left[\begin{array}{cc}
-\frac{1}{R} \mathbf{I}_{3} & {[\mathbf{s}]_{\times}} \\
0 & \mathbf{L}_{\omega}
\end{array}\right]
$$

where $\mathbf{L}_{\omega}$ is given by [6]:

$$
\mathbf{L}_{\omega}=\mathbf{I}_{3}-\frac{\theta}{2}[\mathbf{u}]_{\times}+\left(1-\frac{\operatorname{sinc} \theta}{\operatorname{sinc}^{2} \frac{\theta}{2}}\right)[\mathbf{u}]_{\times}^{2},
$$

with $\operatorname{sinc}(x)=\sin x / x$. The orientation control using $\theta \mathbf{u}$ is completely decoupled from the translational motions. In addition, $\mathbf{L}_{\mathbf{s}_{\mathbf{n}}}$ is an upper block triangular square matrix. This property simplifies the stability analysis given in Section III.

\section{Discussion}

From the set $\mathbf{s}_{\mathbf{n}}=(\mathbf{s}, \theta \mathbf{u})$, we can design another set $\left(\mathbf{s}_{\mathbf{t}}, \theta \mathbf{u}\right)$ which fully decouples the control of the translation from the camera rotational motions. Indeed, if we use

$$
\mathbf{S}_{\mathbf{t}}=\left(\mathbf{s}^{*}-{ }^{c *} \mathbf{R}_{c} \mathbf{s}\right)
$$

where $\mathbf{s}^{*}$ is the desired value of $\mathbf{s}$, we obtain:

$$
\mathbf{L}=\left[\begin{array}{cc}
{ }^{\frac{1}{R}}{ }^{c *} \mathbf{R}_{c} & \mathbf{0} \\
\mathbf{0} & \mathbf{L}_{\omega}
\end{array}\right]
$$

Let ${ }^{\mathbf{c} *} \mathbf{t}_{\mathbf{c}}$ be the relative position of the current camera frame w.r.t. the desired camera frame. We can note that $\mathbf{s}_{\mathbf{t}}=\frac{1}{R}{ }^{\mathbf{c} *} \mathbf{t}_{\mathbf{c}}$ corresponds to a classical 3D visual servoing method up to a scale factor where there is no control of the target in the image [4]. In addition, there is no more a linear relationship between the visual features for translation $\mathbf{s}_{\mathbf{t}}$ and the camera translational velocities $\boldsymbol{v}$. We therefore prefer the set $\mathbf{s}_{\mathbf{n}}=(\mathbf{s}, \theta \mathbf{u})$ which is nearly-linearly linked w.r.t. the camera velocities $\mathbf{v}_{c}$ (see (11)) while enabling control of the target in the image.

\section{STABILITY ANALYSIS TO MODELING ERROR}

Here we suppose that all special marks of each target are visible. In this case the set $\mathbf{s}_{\mathbf{n}}$ can be computed. We use the classical control law

$$
\mathbf{v}_{c}=-\lambda{\widehat{\mathbf{L}_{\mathbf{s}_{\mathbf{n}}}}}^{-1}\left(\mathbf{s}_{\mathbf{n}}-\mathbf{s}_{\mathbf{n}}{ }^{*}\right)
$$

where $\mathbf{v}_{c}$ is the camera velocity sent to the low level robot controller, $\lambda$ is a positive gain, $\widehat{\mathbf{L}}_{\mathbf{s}_{\mathbf{n}}}^{-1}$ is the inverse of an approximation of the interaction matrix related to $\mathbf{s}_{\mathbf{n}}$ and $\mathbf{s}_{\mathbf{n}}^{*}$ is the desired value of $\mathbf{s}_{\mathbf{n}}$. Incorporating an error on the estimation $\widehat{R}$ of $R$ does not affect the set $\mathbf{s}_{\mathbf{n}}$ ( $\mathbf{s}$ and $\theta \mathbf{u}$ are measured without any bias from $\mathbf{o}_{\mathbf{s}}, r, \mathbf{p}_{\mathbf{1}_{\mathbf{s}}}$ and $\mathbf{p}_{\mathbf{2}_{\mathbf{s}}}$ ), but leads to the closed-loop system equation:

$$
\dot{\mathbf{e}}=-\lambda \mathbf{L}_{\mathbf{s}_{\mathbf{n}}}{\widehat{\mathbf{L}_{\mathbf{s}_{\mathbf{n}}}}}^{-1} \mathbf{e}
$$

with $\mathbf{e}=\left(\mathbf{s}-\mathbf{s}^{*}, \theta \mathbf{u}\right)$ and

$$
\widehat{\mathbf{L}_{\mathbf{s}_{\mathbf{n}}}}=\left[\begin{array}{cc}
-\frac{1}{\widehat{R}} \mathbf{I}_{3} & {[\mathbf{s}]_{\times}} \\
0 & \mathbf{L}_{\omega}
\end{array}\right] \text {. }
$$

The asymptotic stability is obtained if $\mathbf{L}_{\mathbf{S}_{\mathbf{n}}}{\widehat{\mathbf{L}_{\mathbf{S}_{\mathbf{n}}}}}^{-1}>0$. We have:

$$
\mathbf{L}_{\mathbf{s}_{\mathbf{n}}}{\widehat{\mathbf{L}_{\mathbf{s}_{\mathbf{n}}}}}^{-1}=\left[\begin{array}{cc}
\frac{\widehat{R}}{R} \mathbf{I}_{3} & \left(-\frac{\widehat{R}}{R}[\mathbf{s}]_{\times}+[\mathbf{s}]_{\times}\right) \mathbf{L}_{\omega}^{-1} \\
0 & \mathbf{I}_{3}
\end{array}\right] .
$$

Since $\mathbf{L}_{\mathbf{S}_{\mathbf{n}}}{\widehat{\mathbf{L}_{\mathbf{S}_{\mathbf{n}}}}}^{-1}$ is a square matrix, its determinant can be calculated:

$$
\operatorname{det}\left(\mathbf{L}_{\mathbf{s}_{\mathbf{n}}}{\widehat{\mathbf{L}_{\mathbf{s}_{\mathbf{n}}}}}^{-1}\right)=(\widehat{R} / R)^{3} .
$$

We have thus

$$
\mathbf{L}_{\mathbf{s}_{\mathbf{n}}}{\widehat{\mathbf{L}_{\mathbf{S}_{\mathbf{n}}}}}^{-1}>0 \Longleftrightarrow \widehat{R}>0 .
$$

This condition is also necessary since if $\widehat{R} \leq 0$ then $\mathbf{L}_{\mathbf{s}_{\mathbf{n}}}{\widehat{\mathbf{L}_{\mathbf{s}_{\mathbf{n}}}}}^{-1} \leq 0$ and the system diverges. The robustness domain w.r.t. modeling error is thus very large: $\widehat{R} \in] 0,+\infty[$. In practice, it means that with an approximated value of the radius of the special sphere, the system still converges as soon as the visibilty and robot joint limits constraints are ensured.

\section{FEATURE COMPUTATION ON CENTRAL PROJECTION CAMERAS}

In this section, we use spherical moments to compute the selected set of features $\mathbf{s}_{\mathbf{n}}=(\mathbf{s}, \theta \mathbf{u})$. We first present the different projection models; then we define spherical moments and show how to measure them from image planes. Finally we show how to compute $\mathbf{s}_{\mathbf{n}}$ using first order spherical moments. 


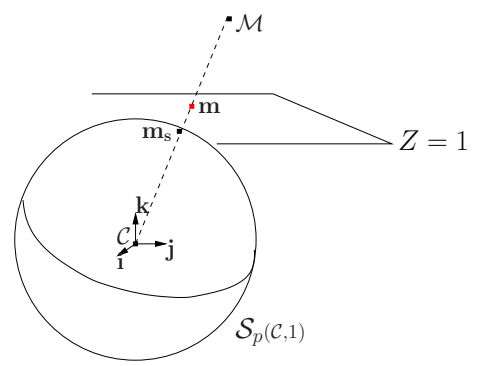

(a)

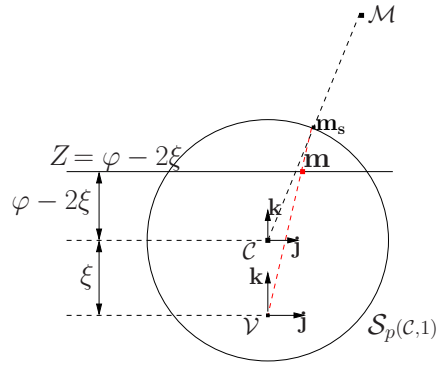

(b)
Fig. 2. Central catadioptric image of a point. (a) Perspective projection $(\varphi=1, \xi=0)$. (b) General case-cut made perpendicular to the image plane.

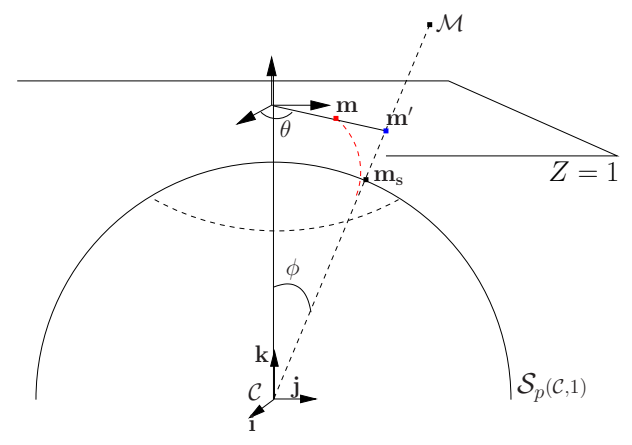

Fig. 3. Fisheye image of a point.

\section{A. Projection models}

1) Central catadioptric cameras: Let $(\varphi, \xi)$ be the mirror parameters of a central catadioptric camera. A unified model in two steps has been proposed for such cameras in [11]. Fig. 2(b) shows the general case of the central catadioptric projection of a feature point $\mathcal{M}$. This model is also valid for a perspective projection model as shown in Fig. 2(a).

Let $\mathcal{F}_{v}$ be the sensor frame $(\mathcal{V}, \mathbf{i}, \mathbf{j}, \mathbf{k})$ and $\mathcal{F}_{c}$ be the center of projection frame $(\mathcal{C}, \mathbf{i}, \mathbf{j}, \mathbf{k})$ where $\mathcal{C}$ is the unique viewpoint of the camera. The first step is the spherical projection of $\mathcal{M}: \mathbf{m}_{\mathbf{s}}=\mathbf{M} /\|\mathbf{M}\|$ where $\mathbf{M}$ is the vector coordinates of $\mathcal{M}$. The point $\mathbf{m}_{\mathbf{s}}$ is then expressed in the sensor frame $\mathcal{F}_{v}$ and projected onto the catadioptric image plane simply as follows:

$$
\mathbf{m}_{x}=\frac{\mathbf{m}_{\mathbf{s} x}}{\mathbf{m}_{\mathbf{s} z}+\xi}, \mathbf{m}_{y}=\frac{\mathbf{m}_{\mathbf{s} y}}{\mathbf{m}_{\mathbf{s} z}+\xi}
$$

where $\mathbf{m}=\left(\mathbf{m}_{x}, \mathbf{m}_{y}\right)$ is the vector coordinates of the image of $\mathbf{m}_{\mathbf{s}}$.

2) Fish-eye cameras: This type of camera does not have a unique viewpoint [18]. An accurate projection model for such cameras has been recently proposed in [12]. The suggested model gives a better approximation of the radial distorsion $\rho(\phi)$ onto the camera image plane:

$$
\mathbf{m}_{x}=\rho(\phi) \cos \theta, \mathbf{m}_{y}=\rho(\phi) \sin \theta
$$

where $\theta=\arctan \left(\mathbf{m}_{y}^{\prime} / \mathbf{m}^{\prime}{ }_{y}\right)$ and $\phi=\arctan \sqrt{\mathbf{m}^{\prime 2}{ }_{x}+\mathbf{m}^{\prime 2}}$ with $\mathbf{m}^{\prime}=\left(\mathbf{m}_{x}^{\prime}, \mathbf{m}_{y}^{\prime}\right)$ the perspective projection of $\mathbf{M}$ (see Fig. 3).

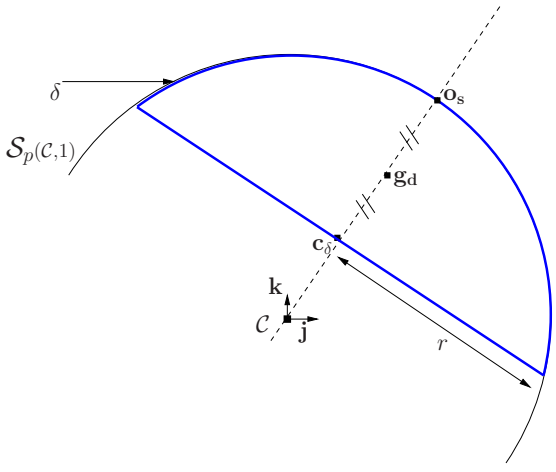

Fig. 4. Dome hat center of gravity $\mathbf{g}_{\mathbf{d}}$.

\section{B. Feature computation using spherical moments}

For both types of camera, it is possible to lift the omnidirectional image of the target onto the surface $\mathcal{S}^{2}$ of the unit sphere $\mathcal{S}_{p}(\mathcal{C}, 1)$; and then to compute the newly designed set of features $\mathbf{s}_{\mathbf{n}}=(\mathbf{s}, \theta \mathbf{u})$ using spherical moments.

1) Spherical moments from the image plane: Sperical moments are useful features for a generic representation of the spherical projection of a target. A spherical moment $m_{p q t}$ can be defined as a geometric moment of the density function $f$ of bi-dimensional spherical distribution:

$$
m_{p q t}=\iint_{\mathcal{D}} f\left(\mathbf{m}_{\mathbf{s} x}, \mathbf{m}_{\mathbf{s} y}, \mathbf{m}_{\mathbf{s} z}\right) \mathrm{d} s
$$

where $\mathcal{D} \subset \mathcal{S}^{2}$ is the surface covered by the spherical projection of the target; and $f\left(\mathbf{m}_{\mathbf{s} x}, \mathbf{m}_{\mathbf{s} y}, \mathbf{m}_{\mathbf{s} z}\right)=\mathbf{m}_{\mathbf{s} x}^{p} \mathbf{m}_{\mathbf{s} y}^{q} \mathbf{m}_{\mathbf{s} z}^{t}$ with $(p, q, t) \in \mathbb{N}^{3}$.

It is possible to compute $m_{p q t}$ on the image plane of any central catadioptric camera:

$$
m_{p q t}=\iint_{\mathcal{R}} f\left(h\left(\mathbf{m}_{x}, \mathbf{m}_{y}\right)\right)\left\|\frac{\partial h}{\partial \mathbf{m}_{x}} \wedge \frac{\partial h}{\partial \mathbf{m}_{y}}\right\| \mathrm{d} \mathbf{m}_{x} \mathrm{~d} \mathbf{m}_{y}
$$

where $\mathcal{R}$ is the surface of the target image on the sensor and $h$ the lifting function given for catadioptric cameras by

$$
h: \begin{array}{ccc}
\mathcal{R} & \rightarrow & \mathcal{D} \\
\left(\mathbf{m}_{x}, \mathbf{m}_{y}\right) & \mapsto & \left(\mathbf{m}_{\mathbf{s} x}, \mathbf{m}_{\mathbf{s} y}, \mathbf{m}_{\mathbf{s} z}\right)
\end{array}
$$

with

$$
\left\{\begin{array}{l}
\mathbf{m}_{\mathbf{s} x}=\frac{\xi+\sqrt{1+\left(1-\xi^{2}\right)\left(\mathbf{m}_{x}^{2}+\mathbf{m}_{y}^{2}\right)}}{\mathbf{m}_{x}^{2}+\mathbf{m}_{y}^{2}+1} \mathbf{m}_{x} \\
\mathbf{m}_{\mathbf{s} y}=\frac{\xi+\sqrt{1+\left(1-\xi^{2}\right)\left(\mathbf{m}_{x}^{2}+\mathbf{m}_{y}^{2}\right)}}{\mathbf{m}_{x}^{2}+\mathbf{m}_{y}^{2}+1} \mathbf{m}_{y} \\
\mathbf{m}_{\mathbf{s} z}=\frac{\xi+\sqrt{1+\left(1-\xi^{2}\right)\left(\mathbf{m}_{x}^{2}+\mathbf{m}_{y}^{2}\right)}}{\mathbf{m}_{x}^{2}+\mathbf{m}_{y}^{2}+1}-\xi .
\end{array}\right.
$$

By expressing (20) and (21) in a spherical coordinates system, it is easy to show that

$$
\left\|\frac{\partial h}{\partial \mathbf{m}_{x}} \wedge \frac{\partial h}{\partial \mathbf{m}_{y}}\right\|=\frac{\left(\xi+\mathbf{m}_{\mathbf{s} z}\right)^{3}}{\xi \mathbf{m}_{\mathbf{s} z}+1} .
$$

where $\mathbf{m}_{\mathbf{s} z}$ is given above. We thus obtain

$$
m_{p q t}=\iint_{\mathcal{R}} \mathbf{m}_{\mathbf{s} x}^{p} \mathbf{m}_{\mathbf{s} y}^{q} \mathbf{m}_{\mathbf{s} z}{ }^{t} \frac{\left(\xi+\mathbf{m}_{\mathbf{s} z}\right)^{3}}{\xi \mathbf{m}_{\mathbf{s} z}+1} \mathrm{~d} \mathbf{m}_{x} \mathrm{~d} \mathbf{m}_{y} .
$$


We can note that when $\xi=0$, (22) exactly corresponds, as expected, to the computation obtained for perspective cameras in [9]:

$$
m_{p q t}=\iint_{\mathcal{R}} \mathbf{m}_{\mathbf{s} x}^{p} \mathbf{m}_{\mathbf{s} y}^{q} \mathbf{m}_{\mathbf{s} z}^{t+3} \mathrm{~d} \mathbf{m}_{x} \mathrm{~d} \mathbf{m}_{y} .
$$

For fish-eye cameras the lifting function $h$ can be obtained using the inverse of the radial distorsion model $\phi(\rho)$ given in [12]:

$$
\left\{\begin{array}{l}
\mathbf{m}_{\mathbf{s} x}=\cos \theta \sin (\phi(\rho)) \\
\mathbf{m}_{\mathbf{s} y}=\sin \theta \sin (\phi(\rho)) \\
\mathbf{m}_{\mathbf{s} z}=\cos (\phi(\rho))
\end{array}\right.
$$

where $\rho=\sqrt{\mathbf{m}_{x}^{2}+\mathbf{m}_{y}^{2}}$ and $\theta=\arctan \left(\mathbf{m}_{y} / \mathbf{m}_{x}\right)$. In this case we have

$$
\left\|\frac{\partial h}{\partial \mathbf{m}_{x}} \wedge \frac{\partial h}{\partial \mathbf{m}_{y}}\right\|=\frac{\sin (\phi(\rho))}{\dot{\rho}(\phi) \rho(\phi)}
$$

from which we deduce

$$
m_{p q t}=\iint_{\mathcal{R}} \mathbf{m}_{\mathbf{s} x}^{p} \mathbf{m}_{\mathbf{s} y}^{q} \mathbf{m}_{\mathbf{s} z}{ }_{z} \frac{\sin (\phi(\rho))}{\dot{\rho}(\phi) \rho(\phi)} \mathrm{d} \mathbf{m}_{x} \mathrm{~d} \mathbf{m}_{y} .
$$

Note that if we do not consider the radial distorsion on the image plane that is $\rho(\phi)=\tan \phi$ (see (19)) we obtain

$$
\left\|\frac{\partial h}{\partial \mathbf{m}_{x}} \wedge \frac{\partial h}{\partial \mathbf{m}_{y}}\right\|=\cos ^{3} \phi=\mathbf{m}_{\mathbf{s} z}^{3}
$$

which exactly corresponds again to the perspective projection case.

2) Feature computation: Now we explain how to compute $\mathbf{s}=\frac{1}{r} \mathbf{o}_{\mathbf{s}}$ using the first order spherical moments $m_{p q t}$ that is for $p+q+t=1$. We recall that the spherical projection of a sphere is a dome hat as shown in Section II-A. Fig. 4 is a zoom on the dome hat where we can see its center of gravity $\mathbf{g}_{\mathbf{d}}$. From Fig. 4 , it is easy to see that $\mathbf{g}_{\mathbf{d}}=\frac{1}{2}\left(\mathbf{c}_{\delta}+\mathbf{o}_{\mathbf{s}}\right)$ where $c_{\delta}=\sqrt{1-r^{2}} \mathbf{o}_{\mathbf{s}}$ is the center of the circular contour $\delta$ given in [8]. Since $\left\|\mathbf{o}_{\mathbf{s}}\right\|=1$, we immediately obtain $r=\sqrt{1-\left(2\left\|\mathbf{g}_{\mathbf{d}}\right\|-1\right)^{2}}$. By noticing that $\mathbf{o}_{\mathbf{s}}=\mathbf{g}_{\mathbf{d}} /\left\|\mathbf{g}_{\mathbf{d}}\right\|$, we finally have

$$
\mathbf{s}=\frac{1}{\left\|\mathbf{g}_{\mathbf{d}}\right\| \sqrt{1-\left(2\left\|\mathbf{g}_{\mathbf{d}}\right\|-1\right)^{2}}} \mathbf{g}_{\mathbf{d}}
$$

where vector coordinates $\mathbf{g}_{\mathbf{d}}$ can be computed from the omnidirectional image plane using the first order spherical moments as follow:

$$
\mathbf{g}_{\mathbf{d} x}=m_{100} / m_{000}, \mathbf{g}_{\mathbf{d} y}=m_{010} / m_{000}, \mathbf{g}_{\mathbf{d} z}=m_{001} / m_{000}
$$

Finally feature $\theta \mathbf{u}$ can be computed from $\mathbf{o}_{\mathbf{s}}, r, \mathbf{p}_{\mathbf{1}_{\mathbf{s}}}$ and $\mathbf{p}_{\mathbf{2}_{\mathbf{s}}}$ as shown in Section II.

To conclude, the selected set of features $\mathbf{s}_{\mathbf{n}}=(\mathbf{s}, \theta \mathbf{u})$ can be computed using spherical moments from the image of $\mathcal{S}_{(\mathcal{O}, R)}$ in the omnidirectional image plane and the images of $\mathcal{P}_{1}$ and $\mathcal{P}_{2}$.

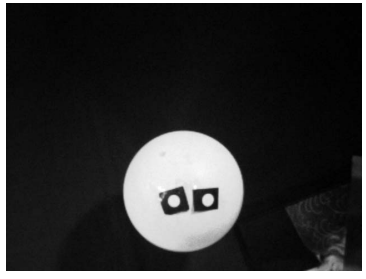

(a)

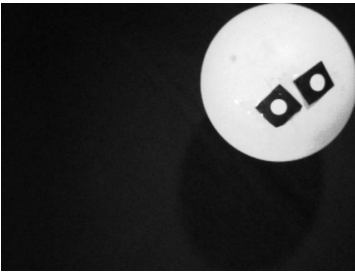

(b)
Fig. 5. Target perspective images. (a) Initial image. (b) Desired image.

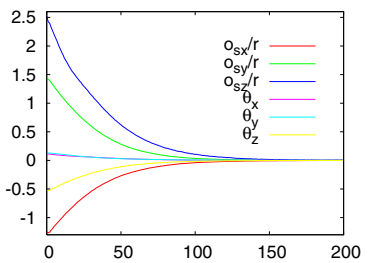

(a) (b)

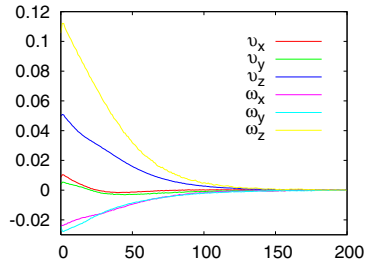

Fig. 6. Ideal case. (a) (s, $\theta \mathbf{u})$ error. (b) Computed camera velocities $(\mathrm{m} / \mathrm{s}$ and $\mathrm{deg} / \mathrm{s}$ ).

\section{RESULTS}

In this section we present the results obtained using either a perspective or a fish-eye camera mounted on the end-effector of a six DOFs gantry manipulator. The target is a white soccer ball of radius $9.5 \mathrm{~cm}$ marked with two points on its surface. The desired set of features $\mathbf{s}_{\mathbf{n}}^{*}$ is measured from the desired image. For all the experiments, the same gain $\lambda=0.1$ has been used.

\section{A. Using a perspective camera}

We first present the results using a perspective camera. Although it is a conventional camera, it has a particular central catadioptric projection model with the mirror parameter $\xi=0$. The initial and desired images are shown in Fig. 5.

1) Ideal case: In the case where the radius of the target is known and we have exact camera calibration values, the behaviour of the system is perfect since $\mathbf{L}_{\mathbf{s}_{\mathbf{n}}}{\widehat{\mathbf{L}_{\mathbf{s}_{\mathbf{n}}}}}^{-1}=\mathbf{I}_{6}$. Fig. 6 pictures the results where we can see, as expected, a nice exponential decrease of the error.

2) Modeling error: Here we validate the robustness to modeling error on the target. The global asymptotic stability of the simple controller (15) has been proved in Section III. We use a radius $\widehat{R}=0.5 R$ in the control scheme. As expected the system still converges as shown by Fig. 7 .

3) Modeling and calibration errors: Let $\left(u_{0}, v_{0}\right)$ be the pixel coordinates of the camera principal point. Let $p_{x}$ (respectively $p_{y}$ ) be the camera focal length on the $x$-axis (respectively $y$-axis). The stability to simultaneously calibration and modeling errors is verified by introducing the following error in the camera intrinsic parameters: $-25 \% p_{x}, 25 \% p_{y}$, $-47 \% u_{0}$ and $20 \% v_{0}$; and by setting the value of the radius of the target to $\widehat{R}=2 R$. Once again the system converges as shown in Fig. 8. 


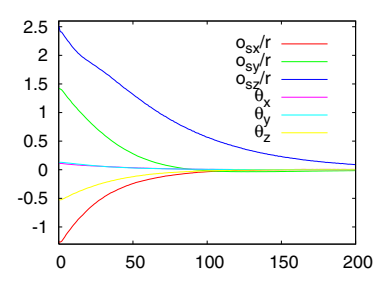

(a)

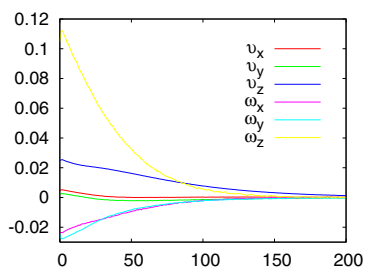

(b)
Fig. 7. Modeling error. (a) (s, $\theta \mathbf{u})$ error. (b) Computed camera velocities $(\mathrm{m} / \mathrm{s}$ and $\mathrm{deg} / \mathrm{s})$.

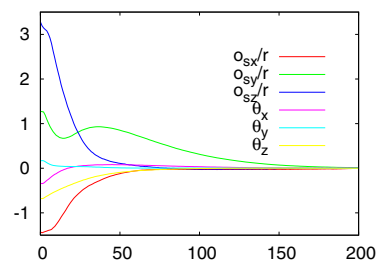

(a)

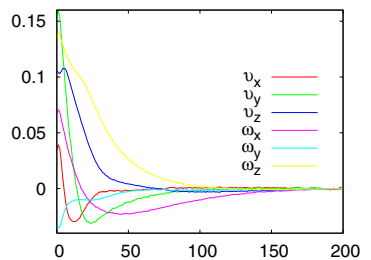

(b)
Fig. 8. Modeling and calibration errors. (a) $(\mathbf{s}, \theta \mathbf{u})$ error. (b) Computed camera velocities $(\mathrm{m} / \mathrm{s}$ and $\mathrm{deg} / \mathrm{s})$.

\section{B. Using a fish-eye camera}

Now we use a fish-eye camera to validate the selected set of features $\mathbf{s}_{\mathbf{n}}=(\mathbf{s}, \theta \mathbf{u})$. More precisely we validate the robustness of the classical control law (15) using w.r.t. to measurement errors. Indeed, the fish-eye spherical moments given by (24) have been estimated using the infinitesimal projected area given by (25) which is a very coarse approximation. Fig. 9 pictures the initial and desired image of the experiment. Here the exact radius of the ball is used. Fig. 10 shows the results where we can see that the system converges. The second shot of the video available at www.irisa.fr/lagadic/demo/demospecial-ball/Demofisheye.avi illustrates this experiment.

\section{CONCLUSION}

In this paper, a spherical projection model has been used for modeling in visual servoing. A sphere marked with two points on its surface has been considered as a target. For this special sphere a new minimal set of six features has been proposed based on a previous work in which the target was a simple sphere. Using the new set, a classical control law has been analytically proven to be asymptotically stable w.r.t. modeling errors. Using the generalization of spherical moments computation from the image of the target, it is possible to measure the new set on most omnidirectional image planes. Finally experimental results with perspective and fisheye cameras have confirmed the validity of the theoretical results.

\section{ACKNOWLEDGMENT}

The authors would like to thank A. Diosi, M. Marey and D. Folio for their help during experiments.

\section{REFERENCES}

[1] S. Hutchinson, G. Hager, and P. Corke, "A tutorial on visual servo control," IEEE Trans. on Robotics and Autom., vol. 12, no. 3, pp. 651670, Oct. 1996.

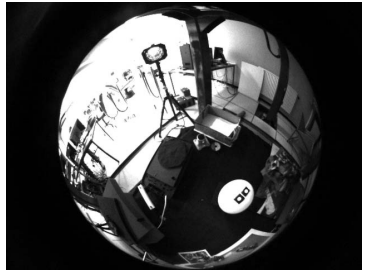

(a)

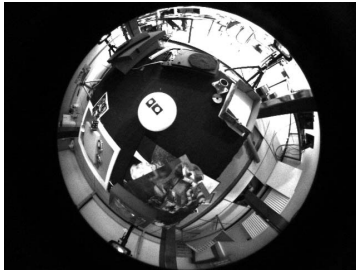

(b)
Fig. 9. Target fish-eye images. (a) Initial image. (b) Desired image.

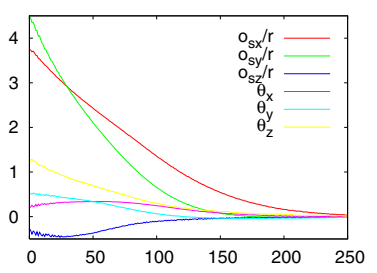

(a)

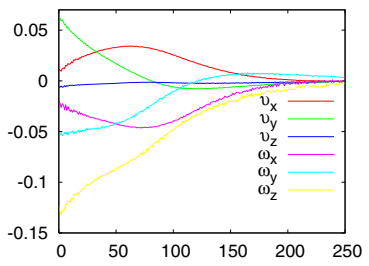

(b)
Fig. 10. System behaviour. (a) $(\mathbf{s}, \theta \mathbf{u})$ error. (b) Computed camera velocities $(\mathrm{m} / \mathrm{s}$ and $\mathrm{deg} / \mathrm{s})$.

[2] F. Chaumette, "Potential problems of stability and convergence in imagebased and position-based visual servoing," in The Confluence of Vision and Control, D. Kriegman, G. Hager, and A. S. Morse, Eds. LNCIS Series, No 237, Springer-Verlag, 1998, pp. 66-78.

[3] W. Wilson, C. Hulls, and G. Bell, "Relative end-effector control using cartesian position-based visual servoing," IEEE Trans. on Robotics and Autom., vol. 12, no. 5, pp. 684-696, Oct. 1996.

[4] L. Deng, W. Wilson, and F. Janabi-Sharifi, "Dynamic perfomance of the position-based visual servoing method in the cartesian and image spaces," in IROS'03, vol. 1, Las Vegas, Nevada, Oct. 2003, pp. 510515.

[5] P. Martinet, J. Gallice, and D. Khadraoui, "Vision based control law using 3d visual features," in World Automation Congress, WAC, vol. 3, Montpellier, France, May 1996, pp. 497-502.

[6] E. Malis, F. Chaumette, and S. Boudet, "2 1/2 d visual servoing," IEEE Trans. on Robotics and Autom., vol. 15, no. 2, pp. 238-250, Apr. 1999.

[7] P. Corke and S. Hutchinson, "A new partitioned approach to imagebased visual visual servo control," IEEE Trans. on Robotics and Autom., vol. 17, no. 4, pp. 507-515, Aug. 2001

[8] R. Tatsambon Fomena and F. Chaumette, "Visual servoing from spheres using a spherical projection model,' in ICRA'07, Rome, Italy, Apr. 2007.

[9] O. Tahri, "Application des moments à l'asservissement visuel et au calcul de pose," Ph.D. dissertation, Université de Rennes 1, Rennes, Mar. 2004.

[10] S. Baker and S. Nayar, "A theory of catadioptric image formation," in ICCV'98, Jan 1998, pp. 35-42.

[11] C. Geyer and K. Daniilidis, "A unifying theory for central panoramic systems and practical implications," ECCV'00, vol. 29, pp. 159-179, May 2000.

[12] J. Kannala and S. Brandt, "A generic camera model and calibration method for conventional, wide-angle and fish-eye lenses," IEEE Trans. on PAMI, vol. 28, no. 8, pp. 1335-1340, 2006.

[13] O. Tahri, F. Chaumette, and Y. Mezouar, "New decoupled visual servoing scheme based on invariants projection onto a sphere," in ICRA'08, Pasadena, CA, May 2008.

[14] T. Hamel and R. Mahony, "Visual servoing of an under-actuated dynamic rigid-body system: an image-based approach," IEEE Trans. on Robotics and Autom., vol. 18, no. 2, pp. 187-198, Apr. 2002.

[15] N. Cowan and D. Chang, "Geometric visual servoing," IEEE Trans. on Robotics, vol. 21, no. 6, pp. 1128-1138, Dec. 2005.

[16] D. Miller and A. Wright, "Autonomous spacecraft docking using multicolor targets," in Proceedings of the 6th Topical Meeting on Robotics, Monterey, CA, February 1995.

[17] B. Espiau, F. Chaumette, and P. Rives, "A new approach to visual servoing in robotics," IEEE Trans. on Robotics and Autom., vol. 8, no. 3, pp. 313-326, Jun. 1992.

[18] M. Born and E. Wolf, Principles of optics. Pergamon Press, 1965. 